\title{
Rosuvastatin for Tocilizumab, an Interleukin-6 Receptor Antibody-induced Dyslipidemia in a Diabetic Patient Complicated With Rheumatoid Arthritis
}

\author{
Hidekatsu Yanaia b, e, Hiroshi Kanekoc, Yuji Hirowarari $^{\mathrm{d}}$
}

\begin{abstract}
Tocilizumab, which blocks interleukin- 6 binding to interleukin-6 receptor, is now approved for the treatment of rheumatoid arthritis (RA). Hyperlipidemia has been reported to be one of the most common adverse effects of tocilizumab, however, the underlying mechanisms remain unknown. To understand lipid metabolism precisely, we measured plasma cytokines, small dense low-density lipoprotein (LDL), oxidized LDL and cholesterol level in each lipoprotein fraction using the high-performance liquid chromatography method before and after the tocilizumab treatment, and also studied the effect of rosuvastatin on tocilizumab-induced dyslipidemia in a diabetic patient complicated with RA. We found that rosuvastatin significantly ameliorated the tocilizumab-induced dyslipidemia.
\end{abstract}

Keywords: Diabetes; Interleukin-6; Small dense low-density lipoprotein; Tocilizumab

\section{Introduction}

Tocilizumab, a newly developed interleukin-6 (IL-6) recep-

\section{Manuscript accepted for publication Oct 12, 2011}

${ }^{\text {a} D e p a r t m e n t ~ o f ~ I n t e r n a l ~ M e d i c i n e, ~ N a t i o n a l ~ C e n t e r ~ f o r ~ G l o b a l ~ H e a l t h ~}$ and Medicine, Kohnodai Hospital, Chiba, Japan

${ }^{\mathrm{b}}$ Clinical Research Center, National Center for Global Health and Medicine, Kohnodai Hospital, Chiba, Japan

${ }^{c}$ Department of Rheumatology, National Center for Global Health and Medicine, Kohnodai Hospital, Chiba, Japan

${ }^{\mathrm{d}}$ Bioscience Division, Tosoh Corporation, Kanagawa, Japan

${ }^{\mathrm{e}}$ corresponding author: Department of Internal Medicine, National

Center for Global Health and Medicine, Kohnodai Hospital, Kohnodai 1-7-1, Ichikawa, Chiba 272-8516, Japan.

Email: dyanai@hospk.ncgm.go.jp

doi:10.4021/jem51w tor monoclonal antibody, used as monotherapy or in combination with methotrexate for rheumatoid arthritis (RA) therapy, leads to a decrease of RA disease activity and an improvement in joint function [1-3]. Although hyperlipidemia has been reported as one of common adverse effects of tocilizumab [1- 3], the precise mechanisms remain unknown. To understand lipid metabolism precisely, we measured plasma cytokines, small dense low-density lipoprotein (LDL) (sdLDL), oxidized LDL (ox-LDL) and cholesterol level in each lipoprotein fraction using the high-performance liquid chromatography (HPLC) method before and after the treatment using tocilizumab [4], and also studied the effect of rosuvastatin on tocilizumab-induced dyslipidemia in a diabetic patient complicated with RA.

\section{Case Report}

A 74-year-old woman developed RA and type 2 diabetes, in 2002 and 2004, respectively. Her RA has been treated by non-steroidal anti-inflammatory drugs and methotrexate (4mg / week), and her diabetes has been treated by dietary therapy. In 2010, the treatment using tocilizumab for RA was introduced because her RA disease activity increased. Her body weight was $51.9 \mathrm{~kg}$ and height $154.0 \mathrm{~cm}$ (BMI $21.9 \mathrm{~kg} /$ $\mathrm{m}^{2}$ ). Fasting plasma glucose and $\mathrm{HbA}_{1 \mathrm{c}}$ levels were $108 \mathrm{mg} /$ $\mathrm{dl}$ and $7.3 \%$, respectively.

After one month of tocilizumab use, serum high-sensitivity C-reactive protein (hs-CRP) significantly decreased and serum adiponectin and interleukin-6 increased (Fig. 1). $\mathrm{HbA}_{1 \mathrm{c}}$ level decreased from 7.3 to $6.8 \%$. Serum levels of total cholesterol (TC) and high-density lipoprotein cholesterol (HDL-C) slightly increased, and LDL-C level decreased, and serum triglyceride (TG) levels significantly increased after the tocilizumab treatment (Fig. 2). In the HPLC study, very low-density lipoprotein cholesterol (VLDL-C) significantly increased and HDL-C decreased (Fig. 3). The sd-LDL and ox-LDL levels were increased (Fig. 4). After two weeks of rosuvastatin use, serum levels of LDL-C, intermediate low-density lipoprotein (IDL)-C, VLDL-C, chylomicron (CM)-C, sd-LDL and ox-LDL were significantly decreased, and HDL-C remarkably increased (Fig. 3, 4). 

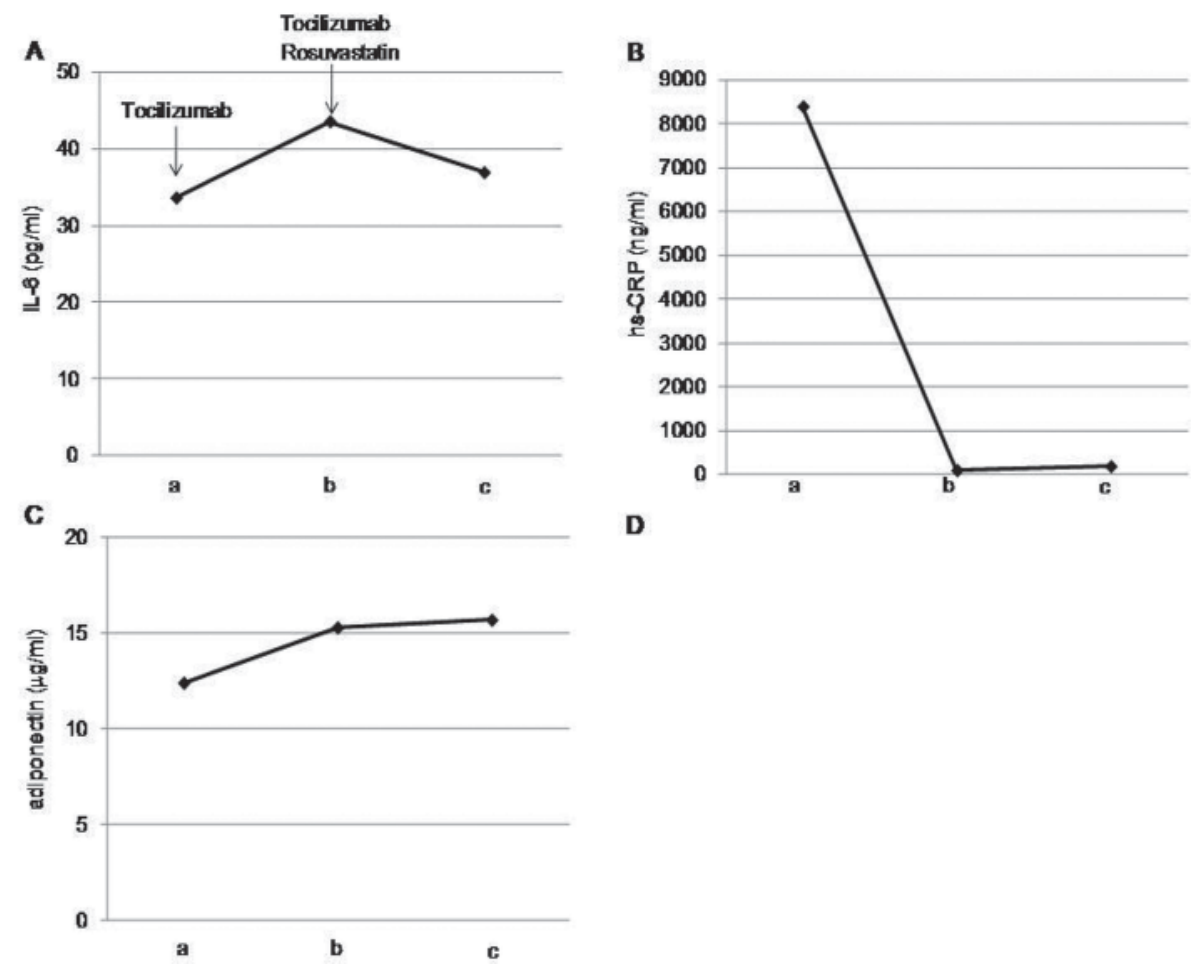

D

Figure 1. Effects of tocilizumab and rosuvastatin on serum interleukin (IL)-6 (A), high-sensitivity C-reactive protein (hs-CRP) (B) and adiponectin (C). a, before the tocilizumab treatment; b, one month after the tocilizumab treatment and before the rosuvastatin use; c, two weeks after the rosuvastatin use.
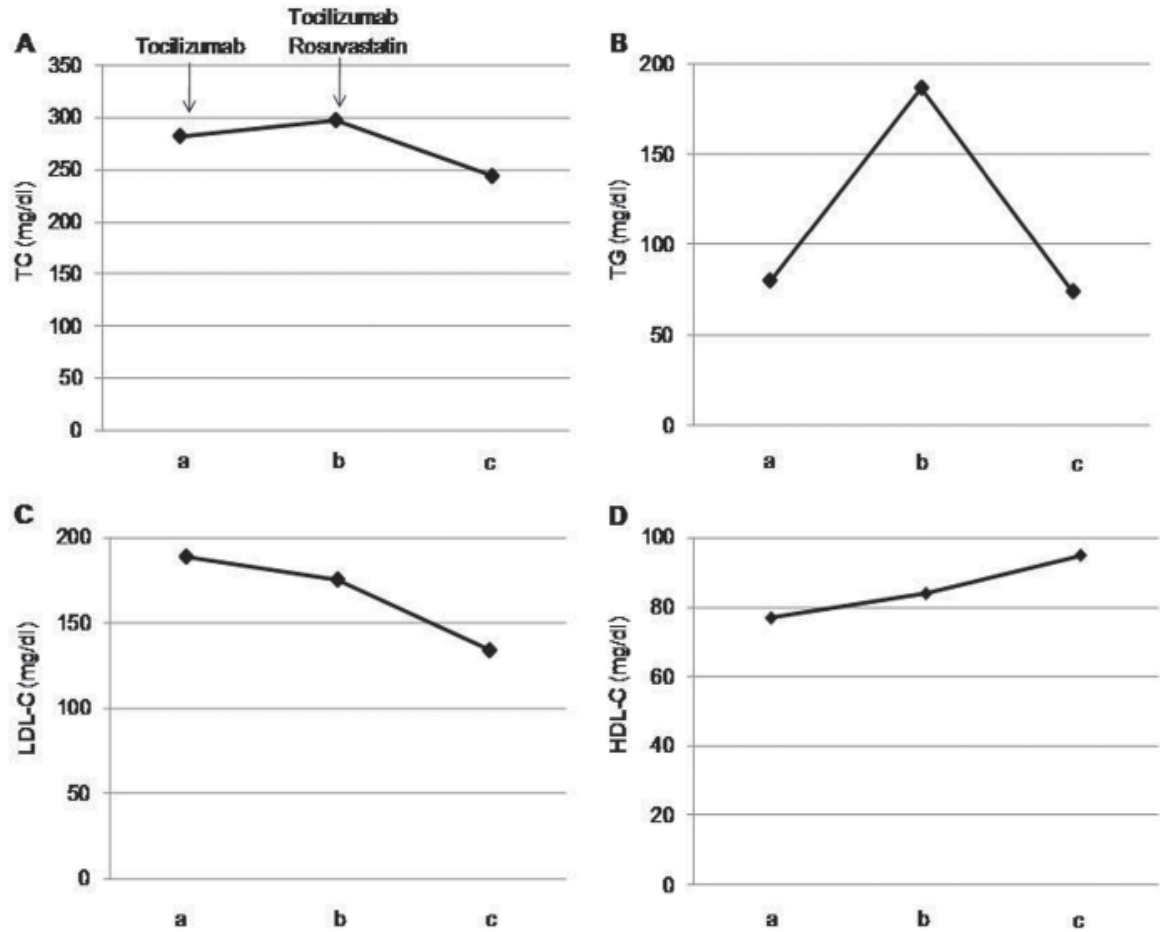

Figure 2. Effects of tocilizumab and rosuvastatin on serum total cholesterol (TC) (A), triglyceride (TG) (B), low-density lipoprotein-cholesterol (LDL-C) (C) and high-density lipoprotein-cholesterol (HDL-C) (D). a, before the tocilizumab treatment; $b$, one month after the tocilizumab treatment and before the rosuvastatin use; $c$, two weeks after the rosuvastatin use. 

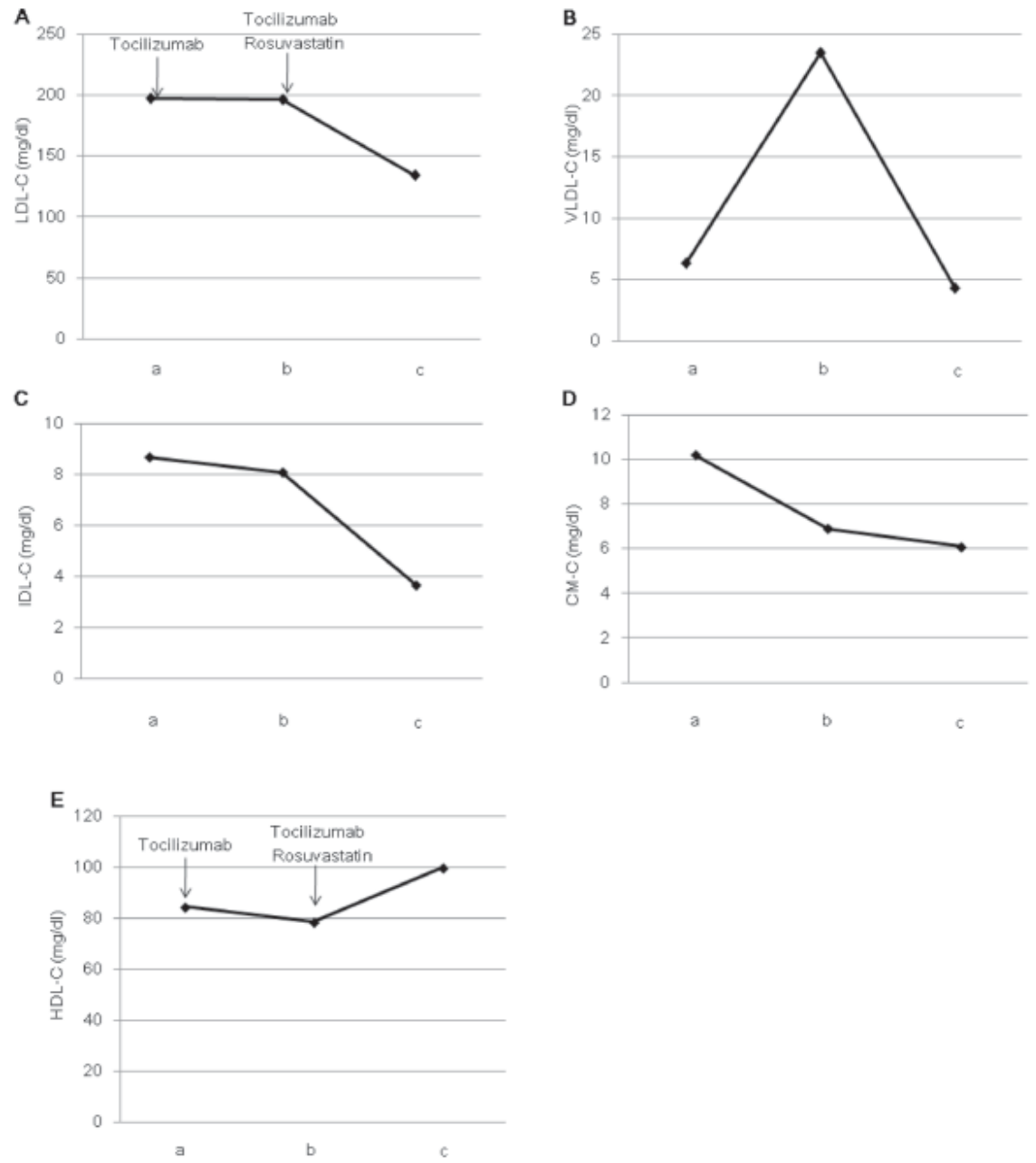

Figure 3. Effects of tocilizumab and rosuvastatin on serum low-density lipoprotein-cholesterol (LDL-C) (A), very low-density lipoprotein-cholesterol (VLDL-C) (B), intermediate-density lipoprotein-cholesterol (IDL-C) (C), chylomicron-cholesterol (CM-C) (D) and high-density lipoprotein-cholesterol (HDL-C) (E), determined by the high-performance liquid chromatography method. $a$, before the tocilizumab treatment; $b$, one month after the tocilizumab treatment and before the rosuvastatin use; c, two weeks after the rosuvastatin use.
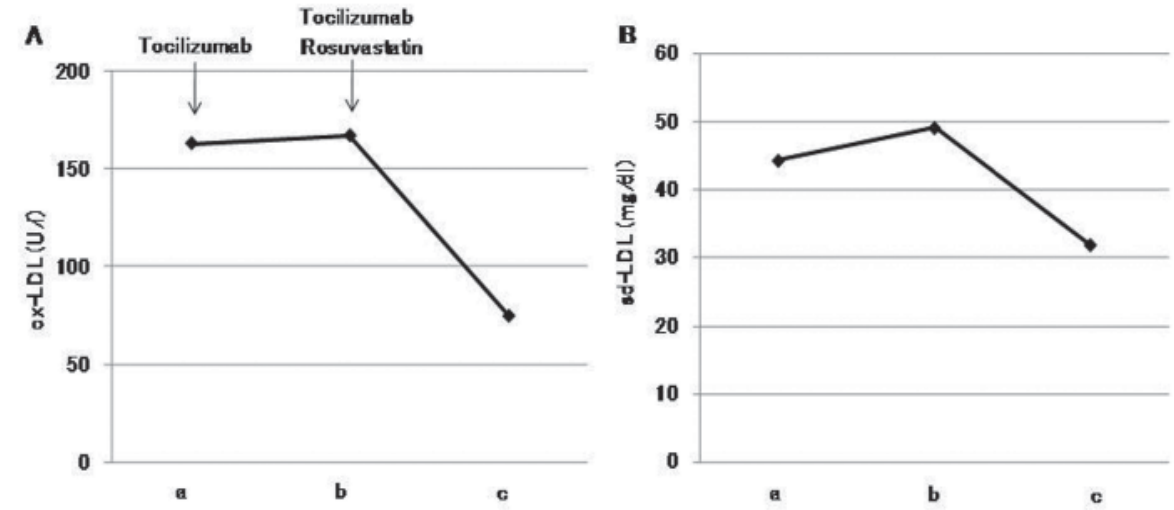

Figure 4. Effects of tocilizumab and rosuvastatin on serum oxidized low-density lipoprotein (ox-LDL) (A) and small dense low-density lipoprotein (sd-LDL) (B). a, before the tocilizumab treatment; b, one month after the tocilizumab treatment and before the rosuvastatin use; c, two weeks after the rosuvastatin use. 


\section{Discussion}

Tocilizumab, which blocks IL-6 binding to IL-6 receptor, is now approved for the treatment of RA [1-3]. In the systematic review of MEDLINE (1998 - June 2008) and International Pharmaceutical Abstracts (1970 - June 2008), hyperlipidemia has been reported to be one of the most common adverse effects of tocilizumab [2]. In another systematic review including eight randomized controlled trial with 3,334 participants, 2,233 treated with tocilizumab and 1,101 controls, significant increases in TC, HDL-C, LDL-C and TG were observed in the tocilizumab treated patients [3]. However, the underlying mechanisms for tocilizumab-induced hyperlipidemia remain unknown.

Tocilizumab decreased hs-CRP and increased adiponectin in our patient, which is favorable for serum lipid metabolism [5, 6], however, tocilizumab remarkably increased TG-rich lipoprotein such as VLDL and atherogenic lipoproteins such as sd-LDL and ox-LDL, and decreased HDL. In our study, serum IL-6 level was increased after the tocilizumab treatment due to the blockade of interleuikin-6 receptor. IL-6 is a cytokine that appears to act as an important systematic regulatory hormone in response to inflammation. Serum IL-6 has been reported to increase in RA patients and to be associated with disease activity of RA [7]. Plasma levels of IL-6 correlate positively with insulin resistance and plasma free fatty acids (FFA) levels [8, 9]. Administration of IL-6 to humans induces elevation of plasma FFA and increased plasma FFA appearance, consistent with increased adipocyte lipolysis of TG $[10,11]$. IL- 6 has been also reported to suppress lipoprotein lipase activity in adipose tissue [12]. Tocilizumab-induced elevation in TG-rich lipoproteins and reduction of HDL may be due to an increased activity of hormone-sensitive lipase and a decreased activity of lipoprotein lipase due to increased serum IL-6 levels by the IL-6 receptor blockade.

Recently, Verges B, et al. reported that rosuvastatin not only induces a significant increase of LDL catabolism, but also has favorable effects on the catabolism of TG-rich lipoproteins, e.g. a significant increase in the catabolism of VLDL and IDL, and a reduction in the production rate of VLDL in type 2 diabetic patients [13]. In our study, rosuvastatin significantly decreased VLDL-C and IDL-C as well as LDL-C, suggesting the effects of rosuvastatin on the metabolism of TG-rich lipoproteins may be beneficial for the tocilizumab-induced dyslipidemia in type 2 diabetic patient. Our study also showed that rosuvastatin increases HDL-C which was reduced by tocilizumab.

Rosuvastatin has been reported to decrease sd-LDL and ox-LDL [14, 15]. Rosuvastatin significantly decreased these elevated atherogenic lipoproteins after the tocilizumab treatment in our patient, which also supports the usefulness of rosuvastatin for tocilizumab-induced dyslipidemia.

\section{Conclusion}

Rosuvastatin significantly ameliorated the tocilizumab-induced dyslipidemia. To confirm our findings, further studies, preferably with larger numbers of subjects, will be needed.

\section{Acknowledgments}

This work was supported by the Grant of National Center for Global Health and Medicine (22-120).

\section{Consent}

Written informed consent was obtained from a patient for publication of this case report.

\section{Authors' Contributions}

All authors were involved in study design, drafting the manuscript and read and approved the final manuscript.

\section{Competing Interests}

The authors declare that they have no competing interests.

\section{References}

1. Ding C, Cicuttini F, Li J, Jones G. Targeting IL-6 in the treatment of inflammatory and autoimmune diseases. Expert Opin Investig Drugs. 2009;18(10):1457-1466.

2. Plushner SL. Tocilizumab: an interleukin-6 receptor inhibitor for the treatment of rheumatoid arthritis. Ann Pharmacother. 2008;42(11):1660-1668.

3. Singh JA, Beg S, Lopez-Olivo MA. Tocilizumab for rheumatoid arthritis. Cochrane Database Syst Rev. 2010(7):CD008331.

4. Hirowatari Y, Yoshida H, Kurosawa H, Doumitu KI, Tada N. Measurement of cholesterol of major serum lipoprotein classes by anion-exchange HPLC with perchlorate ion-containing eluent. J Lipid Res. 2003;44(7):14041412.

5. Jovicic S, Ignjatovic S, Dajak M, Kangrga R, MajkicSingh N. Association of lipid and inflammatory markers with C-reactive protein in cardiovascular risk assessment for primary prevention. Clin Lab. 2009;55(1112):411-419.

6. Kantartzis K, Rittig K, Balletshofer B, Machann J, Schick F, Porubska K, Fritsche A, et al. The relationships of plasma adiponectin with a favorable lipid profile, de- 
creased inflammation, and less ectopic fat accumulation depend on adiposity. Clin Chem. 2006;52(10):19341942.

7. Houssiau FA, Devogelaer JP, Van Damme J, de Deuxchaisnes CN, Van Snick J. Interleukin-6 in synovial fluid and serum of patients with rheumatoid arthritis and other inflammatory arthritides. Arthritis Rheum. 1988;31(6):784-788.

8. Kern PA, Ranganathan S, Li C, Wood L, Ranganathan G. Adipose tissue tumor necrosis factor and interleukin-6 expression in human obesity and insulin resistance. Am J Physiol Endocrinol Metab. 2001;280(5):E745-751.

9. Vozarova B, Weyer C, Hanson K, Tataranni PA, Bogardus C, Pratley RE. Circulating interleukin-6 in relation to adiposity, insulin action, and insulin secretion. Obes Res. 2001;9(7):414-417.

10. van Hall G, Steensberg A, Sacchetti M, Fischer C, Keller C, Schjerling P, Hiscock N, et al. Interleukin-6 stimulates lipolysis and fat oxidation in humans. J Clin Endocrinol Metab. 2003;88(7):3005-3010.

11. Petersen EW, Carey AL, Sacchetti M, Steinberg GR,
Macaulay SL, Febbraio MA, Pedersen BK. Acute IL-6 treatment increases fatty acid turnover in elderly humans in vivo and in tissue culture in vitro. Am J Physiol Endocrinol Metab. 2005;288(1):E155-162.

12. Greenberg AS, Nordan RP, McIntosh J, Calvo JC, Scow $\mathrm{RO}$, Jablons D. Interleukin 6 reduces lipoprotein lipase activity in adipose tissue of mice in vivo and in 3T3-L1 adipocytes: a possible role for interleukin 6 in cancer cachexia. Cancer Res. 1992;52(15):4113-4116.

13. Verges B, Florentin E, Baillot-Rudoni S, Monier S, Petit JM, Rageot D, Gambert P, et al. Effects of $20 \mathrm{mg}$ rosuvastatin on VLDL1-, VLDL2-, IDL- and LDL-ApoB kinetics in type 2 diabetes. Diabetologia. 2008;51(8):13821390.

14. Bahadir MA, Oguz A, Uzunlulu M, Bahadir O. Effects of different statin treatments on small dense low-density lipoprotein in patients with metabolic syndrome. J Atheroscler Thromb. 2009;16(5):684-690.

15. Ono K, Takaishi M. Effects of Rosuvastatin on Oxidized-LDL Cholesterol in the Elderly. J Atheroscler Thromb. 2009;16(2):135-136. 